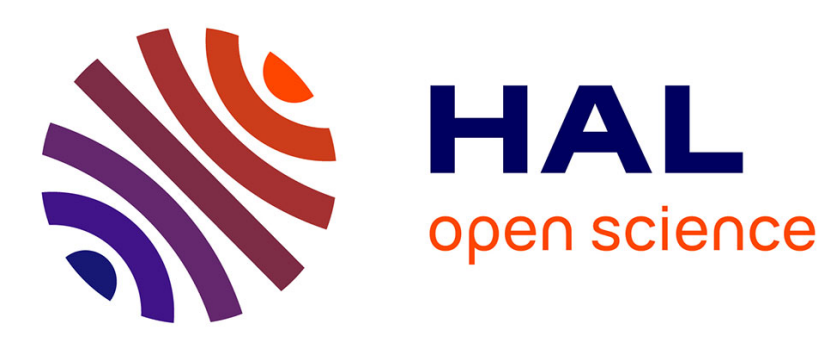

\title{
Modeling the long-range wave propagation by a split-step wavelet method
}

\author{
Hang Zhou, Rémi Douvenot, Alexandre Chabory
}

\section{To cite this version:}

Hang Zhou, Rémi Douvenot, Alexandre Chabory. Modeling the long-range wave propagation by a split-step wavelet method. Journal of Computational Physics, 2020, 402, pp.109042. 10.1016/j.jcp.2019.109042 . hal-02346081

\section{HAL Id: hal-02346081 \\ https://hal-enac.archives-ouvertes.fr/hal-02346081}

Submitted on 5 Nov 2019

HAL is a multi-disciplinary open access archive for the deposit and dissemination of scientific research documents, whether they are published or not. The documents may come from teaching and research institutions in France or abroad, or from public or private research centers.
L'archive ouverte pluridisciplinaire HAL, est destinée au dépôt et à la diffusion de documents scientifiques de niveau recherche, publiés ou non, émanant des établissements d'enseignement et de recherche français ou étrangers, des laboratoires publics ou privés. 


\title{
Modeling the Long-Range Wave Propagation by a Split-Step Wavelet Method
}

\author{
Hang Zhou ${ }^{2,3,1}$, Rémi Douvenot ${ }^{1}$, and Alexandre Chabory ${ }^{1}$ \\ ${ }^{1}$ ENAC, TELECOM-EMA, Université de Toulouse, France \\ ${ }^{2}$ Now with the Sino-European Institute of Aviation Engineering (SIAE), Civil Aviation University \\ of China (CAUC), Tianjin, China \\ ${ }^{3}$ Now with the CAUC-ENAC joint Research Center of Applied Mathematics for Air Traffic \\ Management, Civil Aviation University of China (CAUC), Tianjin, China
}

\begin{abstract}
A split-step wavelet method for simulating the long-range wave propagation is introduced. It is based on the fast wavelet transform. Compared to the split-step Fourier method, this method improves the computation efficiency while keeping a good accuracy. The propagation is performed iteratively by means of a pre-computed matrix containing the individual propagations of the wavelets. A fast computation method of this matrix is also presented. For the radiowave propagation in the low troposphere, a local image method is proposed to account for an impedance ground. Inhomogeneous atmospheres and irregular grounds are also considered. Finally, numerical tests of long-range propagations are performed to show the accuracy and time efficiency of this method.
\end{abstract}

\section{Introduction}

Modeling of the long-range wave propagation is a major issue for numerous applications. The parabolic equation method (PE), introduced by Feit and Fock [1, is considered as one of the most reliable numerical method. It is based on an approximation of the wave equation valid along a paraxial direction and neglecting backward propagation. This method has been widely studied to model the propagation of acoustic waves under the sea, electromagnetic waves in the low atmosphere, or to solve the Schrödinger equation, e.g., [2, 3, 4, 15, 6.

The two mostly-used methods for applying PE are based on either finite-differences (FD) [7] or the split-step Fourier method (SSF) 8. The FD has the advantage of a straightforward implementation of complex boundary conditions. SSF is more numerically efficient because it permits larger grid increments. Consequently, SSF is widely used for long-range wave propagation. The computation is done marching on in distances. At each step, the wave is transformed from the spatial domain to the spectral domain by means of a fast Fourier transform (FFT) along the vertical coordinate.

For electromagnetic propagation in the low troposphere, refractivity, ground boundary condition, and irregular relief can be considered in SSF. The phase-screens method is applied to take into account refractivity in the spatial domain [7]. The discrete mixed Fourier transform [9] includes the reflection over an impedance ground in the spectral transform. Finally, the relief can be considered by different algorithms [7, 10, 11, 12.

Propagation methods in 3D have also been developed with FD and SSF for underwater acoustics [13, 14, 15, 16. These 3D methods model the lateral couplings. For electromagnetic propagation, 3D extensions of SSF have also been developed 17, 18, 19. The 3D method has a high accuracy and the capability to model lateral effects. However, if a large computation domain is considered, the computation burden in time and memory becomes the main concern.

Lately, Zhou et al. have developed the discrete SSF method (DSSF) in both 2D [20, 21] and 3D [22, 21]. This improvement of SSF brings self-consistency as defined by [23, 24. Therefore numerical spurious solutions due to the a-posteriori discretization are avoided. The 2D DSSF is used as the reference in this paper.

Gabor bases/frames 25, 26] decompositions have also been proposed for propagation modeling. They have been combined with analytic formulations of the beam propagation. Gabor-based beam algorithms and frame-based beam summation methods are utilized in various applications involving radiation and scattering in complex environments [26, 27, 28, 29].

Wavelets are widely used for signal processing, numerical analysis and data compression 30. As a Gabor atom, a wavelet is a short length oscillation function that has a space-frequency localization property. In the past twenty years, 
several applications of wavelets in the wave propagation domain have been studied. Wavelets applied to integral equations [31, 32] and time-domain methods [33, 34, 35, 32, have been proposed to improve their computational efficiency. In integral equations in electromagnetics, wavelets are notably used to transform a full-matrix linear system into a sparse one. This latter can be solved efficiently by means of Krylov iterative techniques. However, for simulating the long-range propagation, large computer resources and computation time are still required. Efforts to used wavelets for solving PE [36, 37] have yielded a method as accurate as SSF. However, the computation complexity is the same as SSF since Fourier transforms are used.

In this paper, the split-step wavelet (SSW) method is proposed and applied to electromagnetic waves propagation in the low troposphere. Compared to DSSF, the major difference is that the fast wavelet transform (FWT) 38, 30] is used in place of the FFT, our main purpose being to reduce the computation time. This method is based on a wavelet transform with respect to the vertical coordinate, which has a lower complexity than FFT. Moreover, after a compression step, the wavelet decomposition efficiently yields a sparse representation of a signal. The propagation is performed by a linear combination of the wavelets individually propagated, these individual propagations being stored in a pre-computed matrix. An efficient method for filling the propagation matrix is presented. The atmosphere and relief are accounted in the space domain. In addition, for considering an impedance ground, a local image method is proposed. This method reduces the computational complexity compared to the classical image method.

The article is organized as follows. In section 2, the configuration of the problem is presented. In section 3 , the discrete wavelet transform (DWT) and FWT methods for decomposition and compression are briefly introduced. The choice of the wavelet basis is discussed and an example of wavelet transform and compression is presented. In section 4, the proposed SSW method is comprehensively introduced. Domain truncation, ground condition, irregular atmosphere, and relief are also considered. Then, the computational complexities of SSW and DSSF are compared. Section 5 is devoted to numerical tests demonstrating the accuracy and computational efficiency of SSW.

\section{Configuration and discretization}

In this work, we assume an $e^{j \omega t}$ time-dependence of the fields, where $\omega$ is the angular frequency. The aim is to simulate the propagation in a vertical plane $(x, z)$ with $y$ the direction of invariance. The source is located at $x<0$ and the field $u(x, z)$ is known at $x=0$. The propagation is computed in the region $x>0, z \geq 0$. Any field can be decomposed in a transverse electric (TE) and a transverse magnetic (TM) components with respect to $z$. For the sake of simplicity, only the TE component is presented. The study of the TM component would be similar.

For numerical reasons, the computation domain is discretized and of finite size. The vertical domain is limited to $z \in\left[0, z_{\max }\left[\right.\right.$ and the range is limited to $x \in\left[0, x_{\max }[\right.$. The discretization steps are $\Delta x$ and $\Delta z$. The number of points along the 2 directions are $N_{z}=z_{\max } / \Delta z$ and $N_{x}=x_{\max } / \Delta x$. The following uniform grid is used:

$$
\begin{array}{lll}
x=p_{x} \Delta x & \text { for } & p_{x}=\left\{0, \ldots, N_{x}-1\right\}, \\
z=p_{z} \Delta z & \text { for } & p_{z}=\left\{0, \ldots, N_{z}-1\right\} .
\end{array}
$$

Finally, $u\left[p_{z}\right]$ denotes $u\left(p_{z} \Delta_{z}\right)$. In the following parts, calculations are performed in this discrete domain.

\section{Introduction to the discrete wavelet transform}

In this section, DWT and FWT are briefly introduced. These methods are thoroughly described by Mallat 30]. The choice of the wavelet basis for electromagnetic propagation is justified. Furthermore, a data compression criterion is introduced. Finally, decomposition and compression are applied on an example.

\subsection{Discrete wavelet transform}

The DWT leads to a representation of a discrete function as a linear combination of elementary functions. They are obtained by dilations and translations of a scaling function $\phi$ and a wavelet function $\psi[39$. For a scale $l \in \mathbb{Z}$ and a spatial index $p \in \mathbb{Z}$, the scaling and wavelet functions are

$$
\phi_{l, p}\left[p_{z}\right]=2^{-l / 2} \phi\left[2^{-l} p_{z}-p\right] \quad \text { and } \quad \psi_{l, p}\left[p_{z}\right]=2^{-l / 2} \psi\left[2^{-l} p_{z}-p\right],
$$

respectively.

As proven by Mallat [39], for any $L \in \mathbb{Z},\left(\phi_{L, p}\right)_{p \in \mathbb{Z}}$ and $\left(\psi_{l, p}\right)_{p \in \mathbb{Z}, l \in[1, L]}$ form an orthonormal basis of the space of the finite-energy discrete functions. A discrete function $u$ can thus be represented as the sum of an approximation 
component associated with the scaling function and multiresolution detail components associated with the dilated wavelets functions. The $L$-level multiresolution representation is given by

$$
u\left[p_{z}\right]=\sum_{p \in \mathbb{Z}} a_{L}[p] \phi_{L, p}\left[p_{z}\right]+\sum_{l=1}^{L} \sum_{p \in \mathbb{Z}} d_{l}[p] \psi_{l, p}\left[p_{z}\right],
$$

where $a_{L}[p]$ and $d_{l}[p]$ are the approximation and detail coefficients, respectively.

Due to the finite size of the domain along $z$, for each level $l$, the $p$-indices are limited to $\left[0, N_{p}(l)-1\right]$ with $N_{p}(l)=N_{z} / 2^{l}$. The total number of coefficients is $N_{z}$. For the sake of clarity, in the following, the coefficients are represented by a vector $U$ of dimension $N_{z}$. The double index $(l, p)$ is used to represent the elements of $U$, such that

$$
U_{(l, p)}= \begin{cases}a_{L}[p] & \text { for } \quad l=0, p \in\left[0, N_{p}(L)-1\right], \\ d_{l}[p] & \text { for } \quad l \in[1, L], p \in\left[0, N_{p}(l)-1\right] .\end{cases}
$$

Besides, for the sake of conciseness, scaling functions and wavelets are both designated as wavelets hereafter. Thus, the representation is expressed by

$$
u\left[p_{z}\right]=\sum_{l=0}^{L} \sum_{p} U_{(l, p)} \chi_{l, p}\left[p_{z}\right]
$$

with

$$
\chi_{l, p}\left[p_{z}\right]= \begin{cases}\phi_{L, p}\left[p_{z}\right] & \text { for } \quad l=0 \\ \psi_{l, p}\left[p_{z}\right] & \text { for } \quad l \in[1, L] .\end{cases}
$$

Because of the orthogonality of the basis, these coefficients can be calculated as the inner products of $u$ and the basis elements.

However, to calculate these coefficients in practice, the FWT has been developed by Mallat [39]. This operation has a $\mathcal{O}\left(N_{z}\right)$ complexity [39], to be compared to the complexity of FFT in $\mathcal{O}\left(N_{z} \log _{2}\left(N_{z}\right)\right)$ [40]. The inverse FWT, which is based on the same principle as the FWT, presents the same complexity.

Finally, a data compression is applied on the wavelet representation of the signal, taking advantage of the orthonormality of the basis. By forcing to 0 the coefficients that are weaker than a threshold $V_{\mathrm{s}}$, a regular signal can be approximated by a sparse set of coefficients within a chosen accuracy. After compression, the number of non-zero coefficients is usually much smaller than the original size of the signal $N_{z}$. The vector $U$ obtained after compression, denoted as $\tilde{U}$, is a sparse vector with a maximal root mean square error (RMSE) of $20 \log V_{\mathrm{s}}$.

Note that the choice of the wavelet basis is important for the efficiency of the method. We choose the wavelet family "symlets" of order 6. These wavelets can be used for FWT. Furthermore, they have a compact support, a good regularity, and are almost symmetric [30. Therefore, they are good candidates for the SSW algorithm.

\subsection{Wavelet decomposition and compression of an electromagnetic field}

An example of FWT and compression applied to a field is shown in this section. A 2D complex source point (CSP) [41] with a frequency $f_{0}=300 \mathrm{MHz}$ is considered at $x_{s}=\left(x_{w 0}+j k W_{0}^{2} / 2\right), y_{s}=0 \mathrm{~m}, z_{s}=1000 \mathrm{~m}$, with $x_{w 0}=-50 \mathrm{~m}$ and $W_{0}=5 \mathrm{~m}$. This field is sampled on $N_{z}=2048$ points with $\Delta z=1 \mathrm{~m}$. Its amplitude is plotted in Figure 1 in blue.

The compression rate $(\mathrm{CR})$ is defined as

$$
\mathrm{CR}=\frac{\text { Number of zeros }}{\text { Total number of coefficients }} .
$$

For $L=3, V_{\mathrm{s}}=10^{-3}$, the comparison of the coefficients before and after compression is shown in Figure 2 . The number of non-zero compressed coefficients is 22 , which is much smaller than $N_{z}$. Furthermore, we see that the detail coefficients at level 1 are all set to zero. The signal is only represented by the coefficients of level 2 and 3 . The signal is automatically described by the wavelets at appropriate dilations.

The signal reconstructed from the wavelet coefficients is given in Figure 1. The RMSE between the two signals (original and reconstructed from the wavelets after compression) is $-61.6 \mathrm{~dB}$.

The next section presents how to efficiently propagate this sparse field representation in complex environments. 


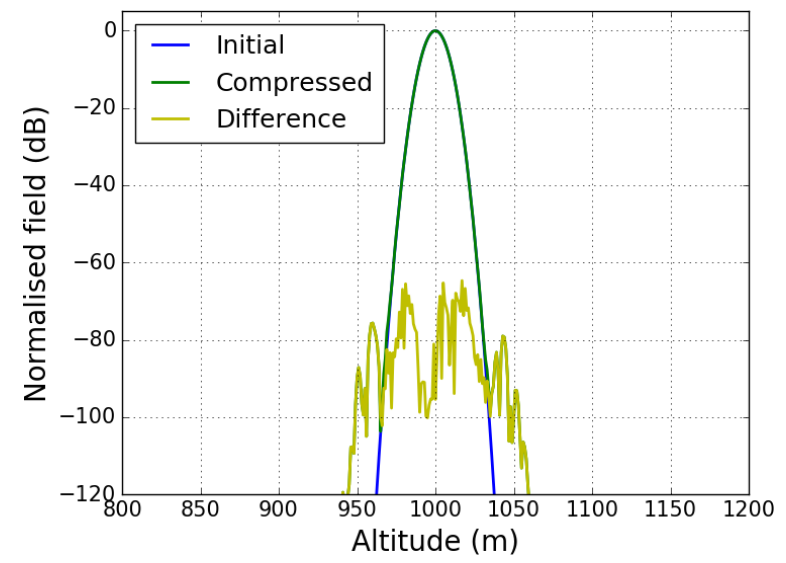

Figure 1: Amplitude of the signal radiated by the CSP.

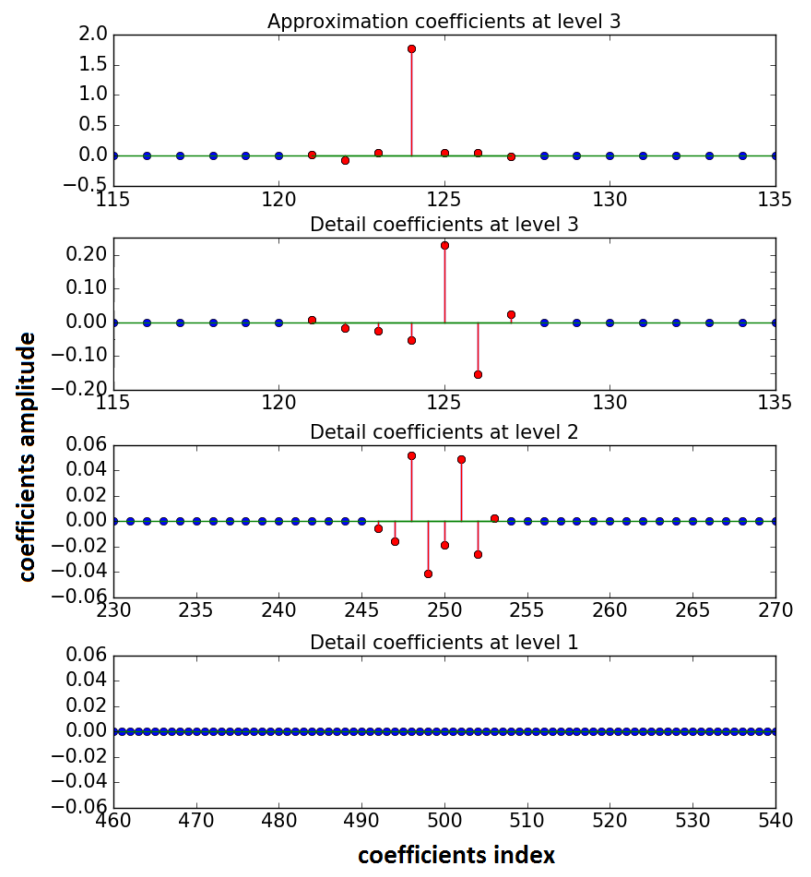

Figure 2: Coefficients of the wavelet decomposition: non-zero coefficients are in red, null coefficients in blue. 


\section{Split-step wavelet method}

Based on the wavelet transform and compression introduced in the previous sections, the SSW method is derived in the present section. The overview of the method is exposed. Then, specific points are addressed: the construction of the propagation matrix, the apodisation, the local image method, the accounting of the relief and atmosphere, and the computational complexity.

\subsection{Overview of the split-step wavelet method}

SSW is performed going back and forth from a spatial to a wavelet representation of the wave so as to model the propagation iteratively at increasing distances. The field is supposed to be known at $x=0$. Then, the propagation from $x$ to $x+\Delta x$, with $x=p_{x} \Delta x$, is simulated step by step as follows:

1. The field $u(x, z)$ is represented as a sparse wavelet vector $\tilde{U}(x)$ after applying FWT (denoted as $W$ ) and a compression with threshold $V_{\mathrm{s}}($ denoted as $C$ ) as introduced in Section III.D, such that

$$
\tilde{U}(x)=C W u(x, z) .
$$

2. The free-space propagation step is considered in the wavelet domain. The coefficients vector $U_{\mathrm{fs}}(x+\Delta x)$ after free-space propagation on a distance $\Delta x$ is given by

$$
U_{\mathrm{fs}}(x+\Delta x)=M \tilde{U}(x),
$$

where $M$ is the pre-computed free-space propagation matrix, detailed in section 4.2 , that models the waveletto-wavelet propagations. This matrix is compressed with a threshold $V_{M}$. The elements of $M$, i.e. $M_{(l, p),\left(l^{\prime}, p^{\prime}\right)}$, satisfy

$$
U_{\mathrm{fs}(l, p)}(x+\Delta x)=\sum_{l^{\prime}, p^{\prime}} M_{(l, p),\left(l^{\prime}, p^{\prime}\right)} \tilde{U}_{\left(l^{\prime}, p^{\prime}\right)}(x) .
$$

3. The field $u_{\mathrm{fs}}(x+\Delta x, z)$ propagated in free space is recomposed by an inverse FWT (denoted as $W^{-1}$ ) from the propagated wavelets.

$$
u_{\mathrm{fs}}(x+\Delta x, z)=W^{-1} U_{\mathrm{fs}}(x+\Delta x) .
$$

4. Apodization, atmosphere, and relief can be applied in the spatial domain as in the classical SSF algorithm [9], here represented by the operator $D$.

As a conclusion, the propagation from $x$ to $x+\Delta x$ is simulated step by step as

$$
u(x+\Delta x, z)=D W^{-1} M C W u(x, z) .
$$

\subsection{Pre-computation of the free-space propagation matrix}

In this section, we present the pre-computation of the matrix $M$. We take into account the translation and dilation properties of the wavelet basis so as to reduce its computation cost. The aim is to obtain $M_{(l, p)\left(l^{\prime}, p^{\prime}\right)}$ for all $l, l^{\prime}, p$, and $p^{\prime}$. The indices $l^{\prime}$ and $l$ correspond to the wavelet levels before and after propagation, respectively. The indices $p^{\prime}$ and $p$ correspond to the positions along the vertical axis before and after propagation, respectively.

The computation of the matrix comprises two steps:

- Wavelet propagation on a distance $\Delta x$ : For the first step, only one wavelet $\chi_{l^{\prime}, 0}$ on level $l^{\prime}$ is considered. It is then propagated on $\Delta x$ using DSSF. (Any other propagation method can be used here.) As illustrated in Figure 3, for any $p^{\prime}$, the propagated wavelets $\chi_{l^{\prime}, p^{\prime}}(\Delta x, z)$ can be deduced from $\chi_{l^{\prime}, 0}(\Delta x, z)$ by means of translations of $p^{\prime}$.

- Calculation of the matrix coefficients: The coefficients $M_{(l, p)\left(l^{\prime}, 0\right)}$ are obtained by inverse FWT and compression. The other ones exploit the translation properties of $M$ given here.

For $l=l^{\prime}$, the number of wavelets on the levels $l$ and $l^{\prime}$ are the same, so a translation of 1 on $p^{\prime}$ results in a translation of 1 on $p$. Thus, $M$ satisfies

$$
M_{(l, p+1)\left(l^{\prime}, p^{\prime}+1\right)}=M_{(l, p)\left(l^{\prime}, p^{\prime}\right)} \quad \text { for } \quad l=l^{\prime} .
$$




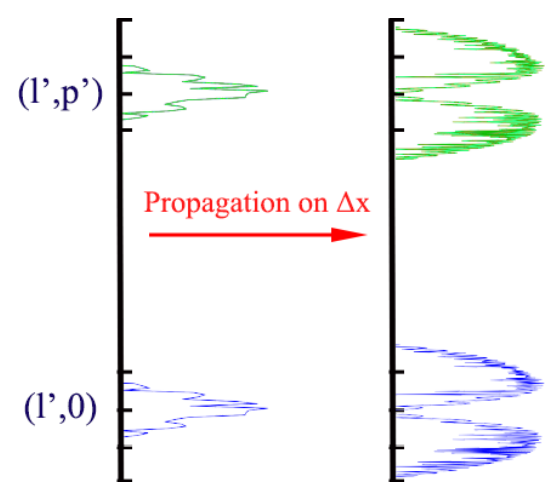

Figure 3: Wavelets at level $l^{\prime}$ propagated on $\Delta x$ on positions 0 and $p^{\prime}$.

Secondly, for $l=l^{\prime}+1$, they are twice as many wavelets on level $l^{\prime}$ than on level $l$. Thus, a translation of 2 on $p^{\prime}$ results in a translation of 1 on $p$. Thus, we have

$$
M_{(l, p+1)\left(l^{\prime}, p^{\prime}+2\right)}=M_{(l, p)\left(l^{\prime}, p^{\prime}\right)} \quad \text { for } \quad l=l^{\prime}+1 .
$$

Finally, for $l=l^{\prime}-1$, they are half as many wavelets on level $l^{\prime}$ than on level $l$. In other words, a translation of 1 on $p^{\prime}$ results in a translation of 2 on $p$. All the even coefficients $p$ are obtained this way. The odd coefficients are obtained from translations of the inverse FWT of $\chi_{l^{\prime}, 1}$. (An additional inverse FWT is required.)

The rule can be generalised for any $l$ and $l^{\prime}$ by

$$
\begin{aligned}
& \text { if } l \leq l^{\prime}, M_{\left(l, p+2^{l^{\prime}-l}\right)\left(l^{\prime}, p^{\prime}+1\right)}=M_{(l, p)\left(l^{\prime}, p^{\prime}\right)}, \\
& \text { if } l>l^{\prime}, M_{(l, p+1)\left(l^{\prime}, p^{\prime}+2^{l-l^{\prime}}\right)}=M_{(l, p)\left(l^{\prime}, p^{\prime}\right)} .
\end{aligned}
$$

These properties are used to efficiently fill the matrix $M$. The complete procedure is given in algorithm 1 .

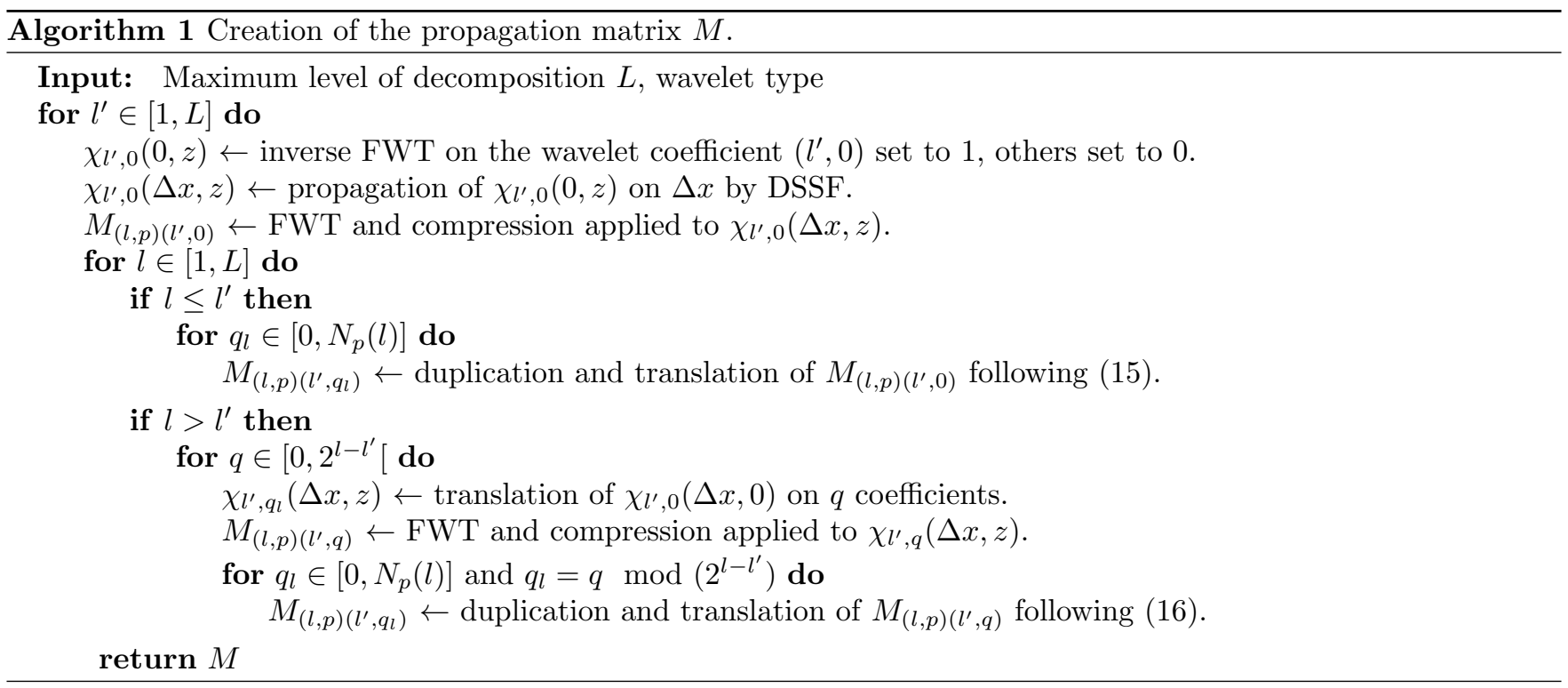

The cases $l=0$ and $l^{\prime}=0$ corresponding to the scaling functions have not been included for the sake on conciseness. They must be treated as additional levels $L$ in both loops. The threshold used for the compressions during the creation of the matrix $M$ is denoted as $V_{M}$.

Finally, only $L+1$ DSSF propagations and $2^{L}$ FWT are necessary to calculate the entire matrix $M$.

\subsection{Domain truncation}

In order to remove spurious reflections over the top boundary and reduce the amount of computation, many methods could be used such as apodization layers, perfectly matched layers, or non-local boundary conditions. In this paper, we use an apodization layer. A Hanning window [7] is applied to the field at the upper half part. 


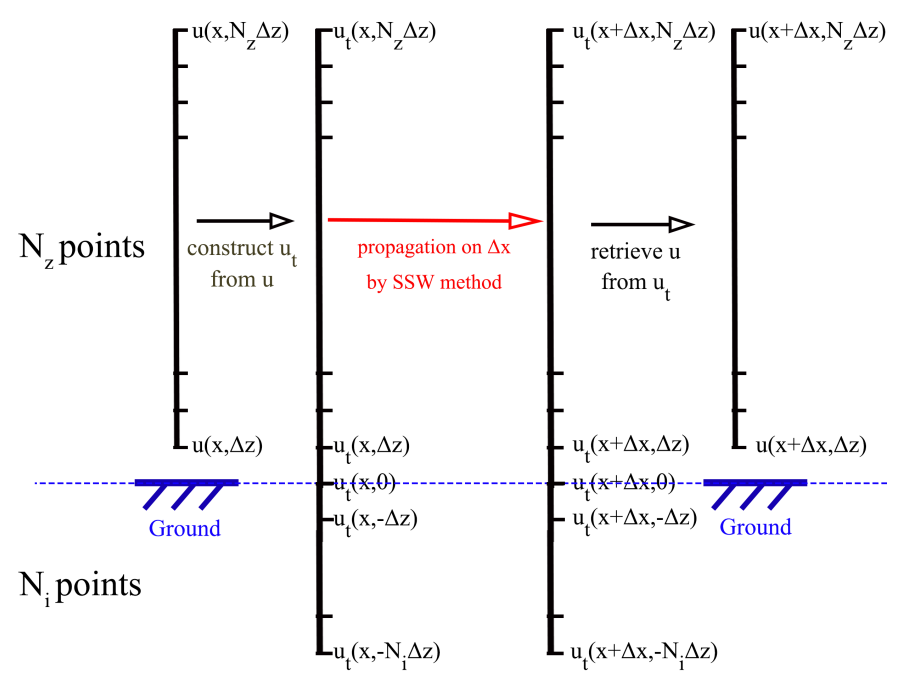

Figure 4: Local perfect electric conductor ground condition for SSW.

\subsection{Ground boundary condition: the local image method}

In DSSF, if an impedance condition is applied at the ground boundary, the Fourier transform is replaced by the discrete mixed Fourier transform 9 . We cannot reproduce this strategy here because the wavelet transform is used.

To consider a perfectly conducting or an impedance ground in SSW, we introduce a local image algorithm. The key idea is to add the symmetric field affected by its reflection coefficient in a thin image layer under the ground level. This field is reset at each propagation step. The local image algorithm is illustrated in Figure 4. The method follows these three steps:

1. At range index $p_{x}$, we know the field $u$ described on $N_{z}$ points. Then it is extended to $u_{t}$ by adding a local image layer under $u$ on $N_{\mathrm{i}}$ points. The choice of $N_{\mathrm{i}}$ is discussed later. In this layer, the field is the image of the upper part. The whole field $u_{t}$ is defined by

$$
u_{t}\left[p_{x}, p_{z}\right]= \begin{cases}u\left[p_{x}, p_{z}\right] & \text { for } p_{z} \in\left[1, N_{z}-1\right], \\ (1+\Gamma) u\left[p_{x}, 0\right] & \text { for } p_{z}=0, \\ \Gamma u\left[p_{x},-p_{z}\right] & \text { for } p_{z} \in\left[-N_{\mathrm{i}},-1\right],\end{cases}
$$

with $\Gamma$ the Fresnel coefficient of the impedance ground, considered as constant at each step. Note here, the ground wave is not considered in this method. Besides, in the case of a PEC ground, $\Gamma=-1$.

2. $u_{t}$ is propagated in free space from $x$ to $x+\Delta x$ by using the SSW propagation strategy.

3. The propagated field $u_{p}\left[p_{x}+1, p_{z}\right]$ is obtained as

$$
u_{p}\left[p_{x}+1, p_{z}\right]=u_{t}\left[p_{x}+1, p_{z}\right], \quad \text { for } p_{z} \in\left[0, N_{z}-1\right] .
$$

The previous algorithm is repeated at each step. As only the image of the field in the close vicinity of the ground is used, we call this algorithm the local image method.

At the bottom of the image layer, no apodization is applied. Therefore, an error appears at the bottom of the domain due to the boundary. However, $N_{\mathrm{i}}$ is chosen so that this error does not reach the domain of interest (i.e., $p_{z} \geq 0$ ). At each step, the field in the image layer is updated using (17). Thus, the error in the image layer is cleared up at each step and never reaches the domain $p_{z} \geq 0$.

$N_{\mathrm{i}}$ is chosen large enough so that the spurious reflections at the bottom of the image domain do not reach the domain $p_{z} \geq 0$ after one computation step. If only propagated waves are accounted $(\Delta z>\lambda / 2)$, the chosen criterion is the maximum width of the wavelets after propagation on $\Delta x$. As the widening of one wavelet on one step $\Delta x$ is much smaller than the total computation domain, $N_{\mathrm{i}} \ll N_{z}$. This makes the local image method computationally efficient. 
Table 1: Computational complexity of SSW and DSSF on one step $\Delta x$.

\begin{tabular}{c||c|c}
\hline & SSW & DSSF \\
\hline wavelet dec. / DFT & $O\left(N_{z}\right)$ & $O\left(N_{z} \log N_{z}\right)$ \\
\hline propagation & $O\left(N_{\mathrm{e}}\right)$ & $O\left(N_{z}\right)$ \\
\hline wavelet rec. / IDFT & $O\left(N_{z}\right)$ & $O\left(N_{z} \log N_{z}\right)$ \\
\hline
\end{tabular}

\subsection{Atmosphere and relief}

To consider a slowly varying atmosphere, the phase screen method 9 is applied to the field. Irregular relief is considered by using a staircase model [7 exactly as for SSF. More precise algorithms [10, 11, 12, could also be applied.

\subsection{Complexity comparison}

Compared to the classical DSSF method, the Fourier transform is replaced by the wavelet decomposition and recomposition. At each range step, the complexity of FFT is $O\left(N_{z} \log N_{z}\right)$, and the complexity of FWT is $O\left(N_{z}+N_{\mathrm{i}}\right) \approx$ $O\left(N_{z}\right)$ because in practice $N_{\mathrm{i}} \ll N_{z}$.

The computation complexity of the propagation step in DSSF is $O\left(N_{z}\right)$. The propagation in SSW follows $(9)$. Since $M$ and $\tilde{U}$ are sparse. The effective multiplication cost $N_{\mathrm{e}}$ is

$$
\begin{aligned}
N_{\mathrm{e}} & =\sum_{(l, p) \in \mathcal{I}_{\mathrm{nz}}(\tilde{U})} \mathcal{N}_{\mathrm{nz}}\left(M_{(l, p),\left(l^{\prime}, p^{\prime}\right)}\right) \\
& \leq \underbrace{\max _{(l, p)}\left(\mathcal{N}_{\mathrm{nz}}\left(M_{(l, p),\left(l^{\prime}, p^{\prime}\right)}\right)\right)}_{N_{M}} \underbrace{\mathcal{N}_{\mathrm{nz}}(\tilde{U})}_{N_{\mathrm{s}}},
\end{aligned}
$$

where $\mathcal{I}_{\mathrm{nz}}$ and $\mathcal{N}_{\mathrm{nz}}$ give the indices and the number of non-zero coefficients in a vector, respectively. $N_{M}$ is the maximum number of non-zero coefficients in one column of $M . N_{\mathrm{s}}$ is the number of non-zero coefficients in $\tilde{U}$. The complexity of this step in SSW is $O\left(N_{\mathrm{e}}\right)$ with $N_{\mathrm{e}} \leq N_{M} N_{\mathrm{s}}$. In practice, $N_{M}$ and $N_{\mathrm{s}}$ are much smaller than $N_{z}$ due to the high compression rate of the wavelet decomposition. The larger the signal and matrix thresholds $V_{\mathrm{s}}$ and $V_{M}$, the smaller $N_{M}$ and $N_{\mathrm{s}}$.

The comparison of the computational complexities of SSW and DSSF is given in Table 1. With a high CR, SSW is faster than DSSF. As the complexities of the algorithms are linear with $N_{x}$, the total complexities are obtained by multiplying by $N_{x}$. Note here, for the creation of the propagation matrix $M$, the complexity of the DSSF propagations is $O\left(L N_{z} \log N_{z}\right)$, and the complexity of the wavelet decompositions is $O\left(2^{L} N_{z}\right)$. They are also negligible for large values of $N_{x}$.

\section{$5 \quad$ Numerical tests}

In this section, several numerical tests are performed to validate the SSW method. A propagation without reflection validates the matrix propagation strategy. Then a simulation with a ground reflection validates the local image method. Finally, a complex case with varying atmosphere and relief is presented. A parametric study to discuss the computation time on this scenario concludes this section. All the simulations are performed on a $1.8 \mathrm{GHz}$ core i7 CPU.

\subsection{Propagation without reflection}

The propagation of a CSP without gound reflection is studied. The aim is to test the accuracy of SSW with different threshold values. The accuracy of SSW is given by comparison to DSSF.

The parameters of the CSP are: frequency $f=300 \mathrm{MHz}, x_{s}=x_{w 0}+j k W_{0}^{2} / 2, y_{s}=0 \mathrm{~m}, z_{s}=2000 \mathrm{~m}$, with $x_{w 0}=-50 \mathrm{~m}$ and $W_{0}=5 \mathrm{~m}$. The computation grid is $x_{\max }=1 \mathrm{~km}, \Delta x=10 \mathrm{~m}, z_{\max }=4096 \mathrm{~m}$, and $\Delta z=1 \mathrm{~m}$. Thus, $N_{x}=100$ and $N_{z}=4096$. The chosen wavelets are symlets 6 , with the maximum level $L=3$.

In order to test the compression thresholds on the matrix $M$ and on the signal, three cases are considered: no compression $\left(V_{M}=V_{\mathrm{s}}=0\right)$, only matrix compression $\left(V_{M}=2 \times 10^{-3}\right.$ and $\left.V_{\mathrm{s}}=0\right)$, and only signal compression $\left(V_{M}=0\right.$ and $\left.V_{\mathrm{s}}=2 \times 10^{-3}\right)$. 


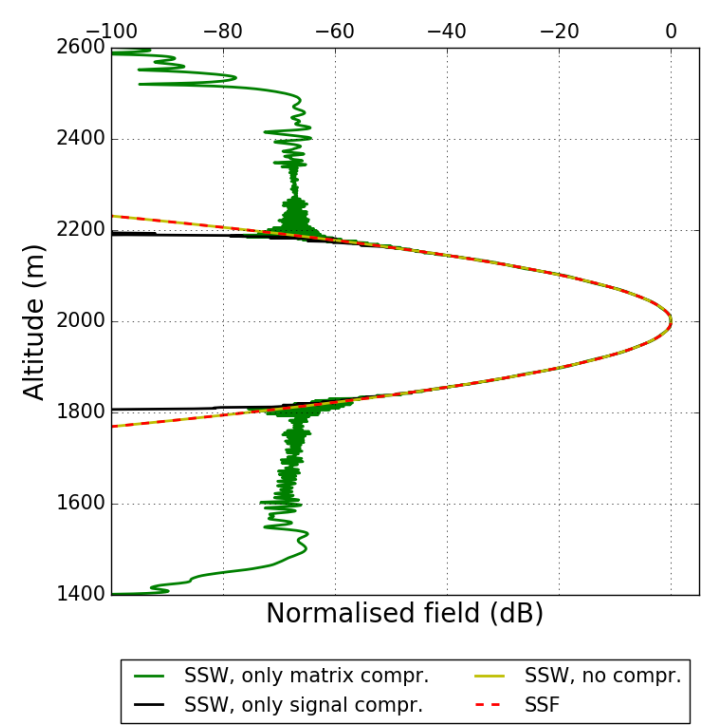

(a) Normalised electric fields (dB) at the maximum range obtained by DSSF (doted line) and SSW (full lines) with different compressions.

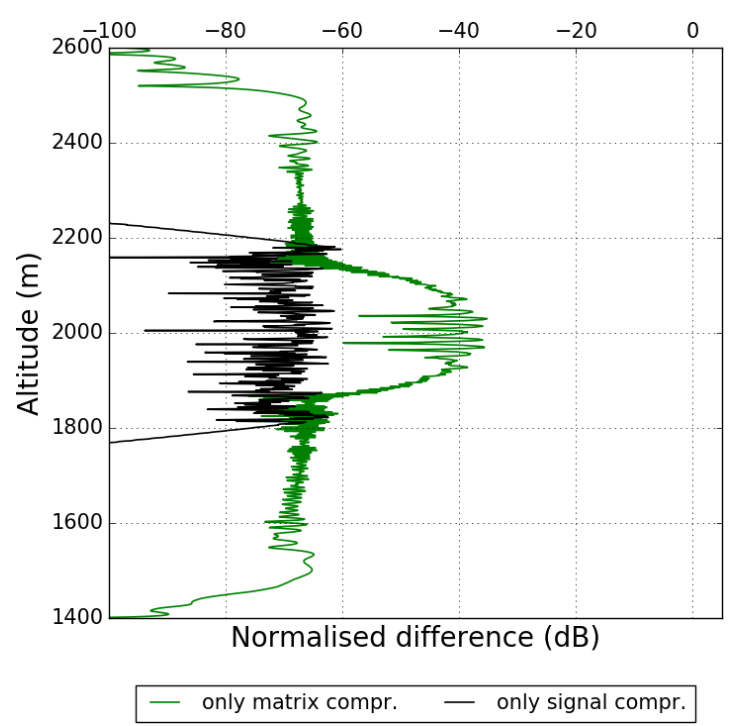

(b) Differences of SSW to DSSF for matrix and signal compressions.

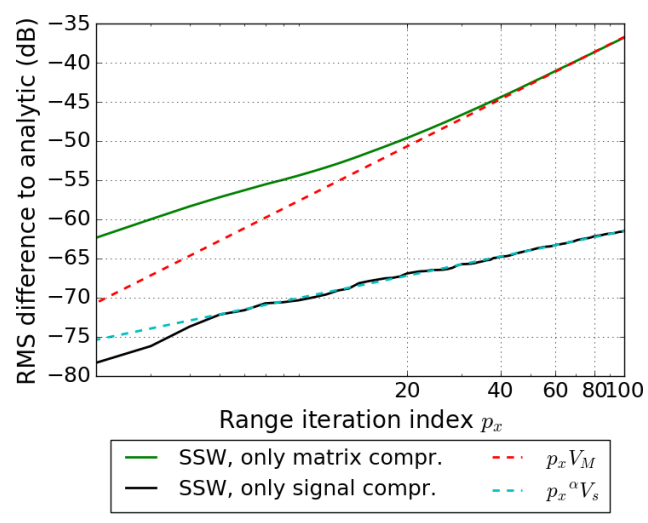

(c) RMS difference (dB) of SSW fields with DSSF at each step in range.

Figure 5: Propagation of a CSP in free-space.

For all cases, the fields at the maximum range and the differences to DSSF are plotted with respect to altitude in Figure $5 \mathrm{a}$ and Figure $5 \mathrm{~b}$, respectively. The fields at the maximum range show a very good agreement. The differences with DSSF are only due to the compression. Without compression, the RMS difference of SSW to DSSF (not visible on Figure $5 \mathrm{~b}$ is $-165.4 \mathrm{~dB}$.

The evaluation of the RMS difference normalised at each step in range is plotted in Figure $5 \mathrm{c}$ in logarithmic scale. For $V_{M} \neq 0$ and $V_{\mathrm{s}}=0$ (green line), the error accumulates in range. The error yielded by the compression on $M$ is of order of $V_{M} N_{x}$ (red dashed line). In the cases $V_{M}=0$ and $V_{\mathrm{s}} \neq 0$ (black line), the error increases in range. However, it is much lower than $V_{\mathrm{s}} N_{x}$ (about $-23 \mathrm{~dB}$ smaller at the maximum range). A least squares fit yields to an error proportional to $V_{\mathrm{s}} N_{x}^{\alpha}$, with $\alpha \approx 0.3$. Consequently, $V_{\mathrm{s}}$ can be chosen much larger than $V_{M}$ in practice. Note that this specific value for $\alpha$ only stands for simple scenarios, when no ground is accounted. Many simulations with different configurations have led to a coefficient $\alpha$ always lower than 0.5 . Therefore, the error due to the signal compression is considered of the order of $V_{\mathrm{s}} N_{x}^{0.5}$. A demonstration of this empirical result has not been obtained yet.

\subsection{Propagation over a planar impedance ground in a homogeneous atmosphere}

The aim of this section is to test SSW with the local image method described in Section 4.4 and to confirm the range dependency of the compression error.

The same source as the previous test is chosen with a height of $z_{s}=30 \mathrm{~m}$. The simulation parameters are 


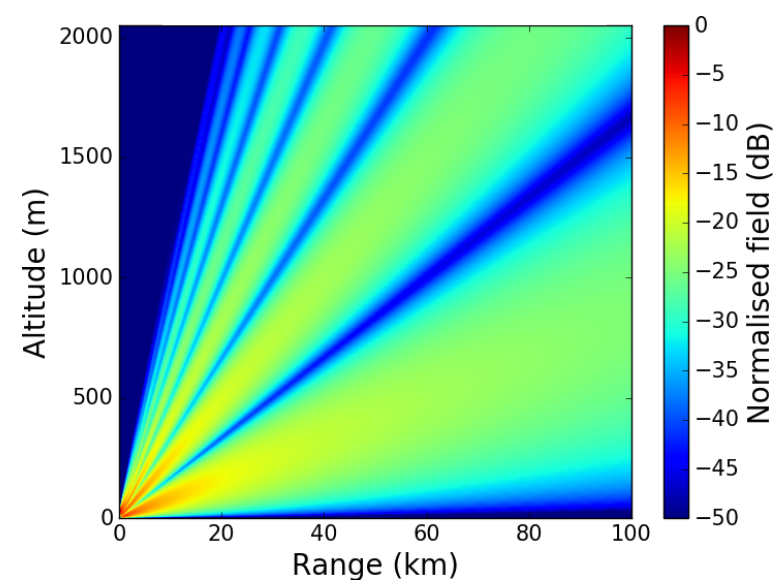

(a) Normalised electric field (dB) in the vertical plane obtained with SSW.

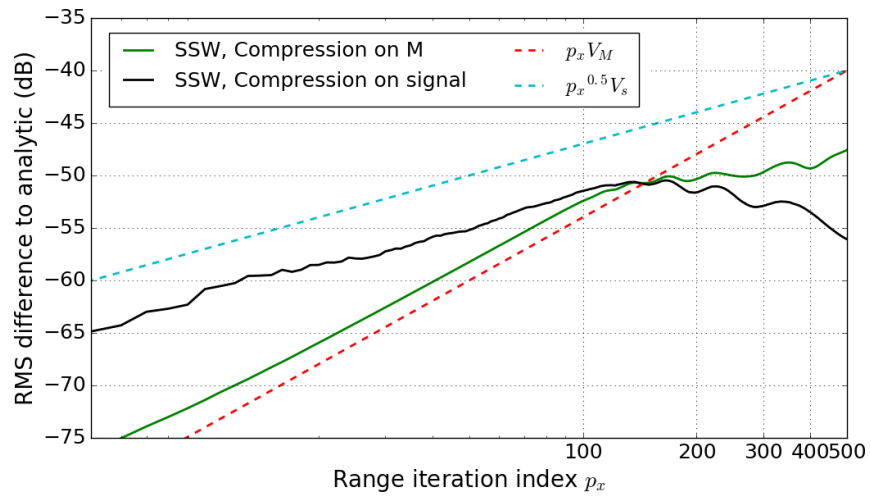

(b) RMS difference (dB) of SSW fields with DSSF at each step in range.

Figure 6: Propagation of a CSP with reflections over an impedance ground.

$x_{\max }=100 \mathrm{~km}, \Delta x=200 \mathrm{~m}, z_{\max }=4096 \mathrm{~m}$, and $\Delta z=1 \mathrm{~m}$. Thus, $N_{x}=500$ and $N_{z}=4096$. A planar impedance ground with $\epsilon_{r}=20$ and $\sigma=0.02 \mathrm{~S} / \mathrm{m}$ is considered. A thin image layer $N_{\mathrm{i}}=200$ is added to the computation domain for applying the local image method.

SSW with compressions on both $M$ and the signal is tested. The expected error $V_{\mathrm{E}}$ on the field at the final range corresponds to the sum of both the compressions, such that $V_{\mathrm{E}}=V_{M} N_{x}+V_{\mathrm{s}} N_{x}^{\alpha}$. The chosen thresholds are $V_{M}=2 \times 10^{-5}$ and $V_{\mathrm{s}}=4.47 \times 10^{-4}$ for the errors due to both compressions to be the same, equal to $-40 \mathrm{~dB}$. Thus, the total expected error is of the order of $-34.0 \mathrm{~dB}$.

The propagated field along range and altitude is shown in Figure 6a, where the interference pattern due to the ground reflection is visible. During the simulation, the CR of the propagation matrix is $86.4 \%$ and the average $\mathrm{CR}$ of the signal is $74.2 \%$. The RMS difference to DSSF on the last vertical is $-47.3 \mathrm{~dB}$. This RMS difference is $-13.3 \mathrm{~dB}$ better than the expected value.

To explain this difference, the error to DSSF with respect to distance is plotted in Figure 6b with compression on the matrix only (in green) and with compression on the signal only (in black). For both cases, the expected final error is $-40 \mathrm{~dB}$. When some energy goes out of the computation domain, after 100 iterations corresponding to a distance of $20 \mathrm{~km}$, the error increase is slower. This may be explained by the fact that some error is removed in the apodization layer. Thus, the actual error due to compression on the last vertical is usually less than the expected error $V_{\mathrm{E}}$.

Finally, the computation time of SSW is $3.2 \mathrm{~s}$ whereas the DSSF simulation lasts $8.6 \mathrm{~s}$. Therefore, SSW is faster than DSSF. Besides, the computation time for creating $M$ is $1.2 \mathrm{~s}$. As a conclusion, SSW with an impedance ground is successfully tested in terms of accuracy and shows its advantage concerning the computation time.

\subsection{Long-range propagation over an impedance irregular relief in an inhomogeneous atmosphere}

In this section, we test a long-range propagation over an irregular relief in an inhomogeneous atmosphere.

In the vertical direction, a surface-based duct is considered. It is modelled by a trilinear modified refractivity profile, as illustrated in Figure 7a. The duct parameters are: $M_{0}=330$ M-units, $z_{b}=100 \mathrm{~m}, z_{t}=200 \mathrm{~m}$, $z_{\max }=4096 \mathrm{~m}$, with gradients $c_{0}=0.118 \mathrm{M}$-units $/ \mathrm{m}, c_{2}=-0.1 \mathrm{M}$-units $/ \mathrm{m}$.

The relief is chosen as 2 small triangular hills of height $100 \mathrm{~m}$ and $200 \mathrm{~m}$. The relief is displayed in Figure $7 \mathrm{~b}$, The characteristics of the impedance ground are chosen as $\epsilon_{r}=20$ and $\sigma=0.02 \mathrm{~S} / \mathrm{m}$.

In this test, the propagation range is $x_{\max }=100 \mathrm{~km}$. The range step is $200 \mathrm{~m}$, giving $N_{x}=500$. We choose the same compression thresholds $V_{M}=2 \times 10^{-5}$ and $V_{\mathrm{s}}=4.47 \times 10^{-4}$. That yields an expected error of the order of $-34.0 \mathrm{~dB}$.

The normalised field propagated using SSW in the vertical plane is shown in Figure $7 \mathrm{c}$ The refractive effects of the surface-based duct and the reflection over the irregular ground are properly simulated. The fields at the maximum range and the difference to DSSF are plotted in Figure 7d. The RMSE of the final field of SSW to DSSF is $-42.0 \mathrm{~dB}, 8 \mathrm{~dB}$ below the expected error. Therefore, SSW works well for a long range simulation over an irregular 


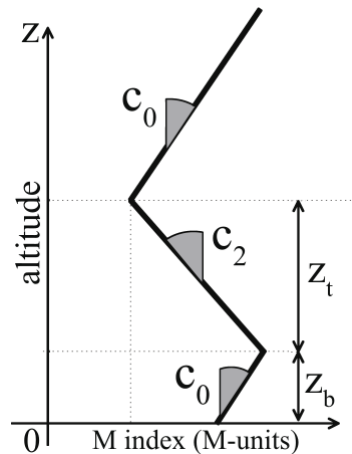

(a) Trilinear model of refractivity.

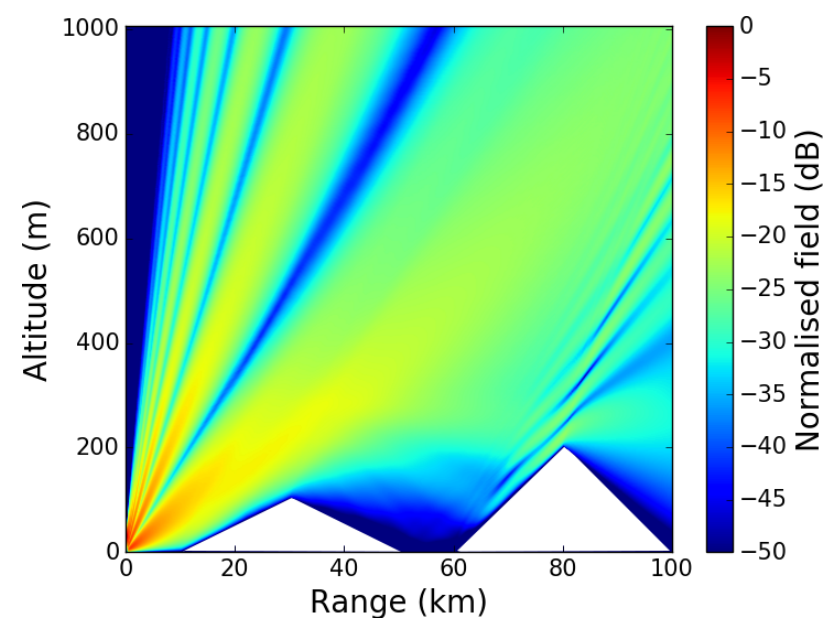

(c) Normalised electric field (dB) obtained by SSW.

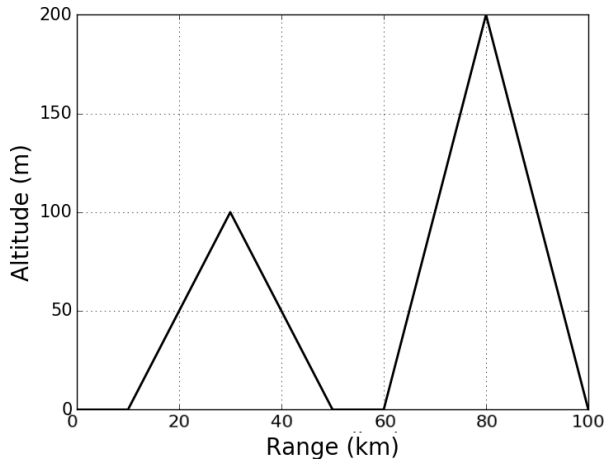

(b) A relief with 2 small triangular hills of heights $100 \mathrm{~m}$ and $200 \mathrm{~m}$.

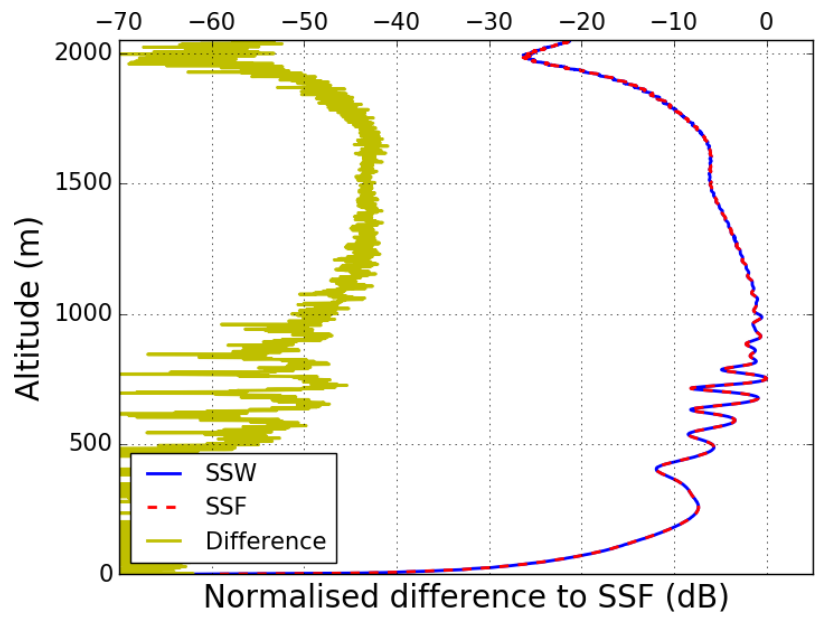

(d) Normalised electric fields $(\mathrm{dB})$ at the maximum range obtained by DSSF and SSW and the difference SSW-DSSF.

Figure 7: Propagation over a planar impedance ground in an inhomogeneous atmosphere and over an irregular relief.

relief in an inhomogeneous atmosphere. As for the previous vase, the computation time is $3.2 \mathrm{~s}$ for SSW and is $8.6 \mathrm{~s}$ for DSSF. SSW has a better computation efficiency than DSSF, even on complex scenarios.

\subsection{Discussion about the computation time}

As stated in Section 4.6, the complexity of SSW depends on the number of vertical points and on the compression rates applied to both the propagation matrix and the signal at each distance step. In this section, this complexity is illustrated. We use the simulation setup of Section 5.3. All else being equal, the vertical number of points $N_{z}$ or the compression rates are varied.

The chosen values and the corresponding computation times obtained with DSSF and SSW are summarized in Table 2. If no compression is applied $\left(V_{M}=0\right.$ and $\left.V_{\mathrm{s}}=0\right)$, SSW is much slower than DSSF. Indeed, even if the wavelet transform is faster than the Fourier transform, the propagation step requires a full matrix mutliplication in the absence of compression.

If smaller compression thresholds are chosen with SSW, some computation time can be saved. Following Section 5.1. the choices $V_{M}=2 \times 10^{-4}$ and $V_{\mathrm{s}}=4.47 \times 10^{-3}$ lead to an acceptable expected error of $-16 \mathrm{~dB}$. The actual simulation gives a computation time shortened by a factor of 2 compared with the previous section and a final RMSE with respect to DSSF of $-22.2 \mathrm{~dB}$.

When $N_{z}$ is greater, the computation time increases less with SSW than with DSSF, which is consistent with the complexities of Table 1 More optimised codes would certainly match better the theoretical complexities. Finally, note that the multiplication cost $\mathcal{O}\left(N_{\mathrm{e}}\right)$ in SSW could still be reduced because the sparse matrix-vector multiplication we use does not take advantage of the sparsity of the vector yet. 
Table 2: Computation times for varying $N_{z}$ and compression thresholds.

\begin{tabular}{|c|c|c|c|c|}
\hline$V_{M}$ & $V_{\mathrm{s}}$ & $N_{z}$ & $\begin{array}{l}\text { comp. time } \\
\text { DSSF (s) }\end{array}$ & $\begin{array}{l}\text { comp. time } \\
\text { SSW (s) }\end{array}$ \\
\hline 0 & 0 & 4096 & 8.6 & 21.1 \\
\hline $2 \times 10^{-4}$ & $4.47 \times 10^{-3} \mathrm{~s}$ & 4096 & 8.6 & 2.0 \\
\hline $2 \times 10^{-5}$ & $4.47 \times 10^{-4} \mathrm{~s}$ & 1024 & 2.2 & 0.9 \\
\hline $2 \times 10^{-5}$ & $4.47 \times 10^{-4} \mathrm{~s}$ & 8192 & 34.6 & 8.0 \\
\hline
\end{tabular}

\section{Conclusion}

The split-step wavelet method (SSW) for the simulation of long-range electromagnetic wave propagation has been introduced. This method is based on the discrete wavelet transform and the discrete electromagnetic theory.

First, the discrete wavelet transform (DWT) and the fast wavelet transform (FWT) methods have been briefly introduced, and the choice of the wavelet family has been discussed.

Then, the proposed SSW method has been comprehensively introduced. The field is represented as a sparse set of coefficients after applying FWT and thresholding. It is then propagated in free-space by means of a pre-computed propagation matrix. The propagated field is recomposed by inverse FWT from the propagated wavelets. Relief, atmosphere, and apodisation are treated in the same way as in split-step Fourier (SSF) techniques. The total field is obtained iteratively.

The strategy to efficiently create the pre-computed propagation matrix has been introduced. Finally, to consider a perfectly conducting or an impedance ground condition, a local image method has been proposed. It is based on the localisation property of the wavelets and does not significantly increases the computation burden.

The computation complexity of SSW has been compared to the discrete SSF (DSSF). SSW is shown to be more efficient than DSSF due to the high compression rate of the wavelet decomposition and the low complexity of the fast wavelet transform.

Finally, numerical tests of wave propagation have been presented to show the accuracy and efficiency of this method. First, propagation in free-space with different thresholds have been tested and compared. Tests of propagation over an impedance ground with both matrix and signal compressions have been performed. We have shown that the error due to compression can be anticipated. Finally, a long-range propagation over an irregular relief in an inhomogeneous atmosphere has been performed. The result of SSW shows a very good match with DSSF, and the simulation parameters are varied to illustrate the complexity of SSW. In conclusion, SSW works well for a long-range simulation with reduced computation time.

Even if the choice of the symlets 6 wavelets has been carefully thought, an exhaustive comparison of the wavelets could lead to a more efficient choice. Moreover, the computation time could be shortened by reducing the size of the pre-computed data and improving the top boundary condition. A theoretical study to obtain an upper bound of the compression error is also necessary. Finally, the method will be extended to 3D configurations in future works.

\section{Acknowledgements}

This work was supported by the Fundamental Research Funds for the Central Universities, project number 3122016 U001.

\section{References}

[1] M. D. Feit, J. A. Fleck, Light propagation in graded-index optical fibers, Applied Optics 17 (24) (1978) 39903998. doi:10.1364/ao.17.003990.

[2] D. F. St Mary, D. Lee, G. Botseas, A modified wide angle parabolic wave equation, Journal of Computational Physics 71 (1987) 304-315. doi:10.1016/0021-9991(87)90032-5.

[3] J. R. Kuttler, G. D. Dockery, Theoretical description of the parabolic approximation/Fourier split-step method of representing electromagnetic propagation in the troposphere, Radio Science 26 (1991) 381-393. doi:10. 1029/91rs00109.

[4] D. Lee, A. D. Pierce, E.-C. Shang, Parabolic equation development in the twentieth century, Journal of Computational Acoustics 8 (2000) 527-637. doi:10.1142/s0218396x00000388 
[5] T. R. Taha, M. I. Ablowitz, Analytical and numerical aspects of certain nonlinear evolution equations. II. Numerical, nonlinear Schrödinger equation, Journal of Computational Physics 55 (1984) 203-230. doi:10. 1016/0021-9991(84)90003-2.

[6] G. M. Muslu, H. A. Erbay, Higher-order split-step Fourier schemes for the generalized nonlinear Schrödinger equation, Mathematics and Computers in Simulation 67 (2005) 581-595. doi:10.1016/j.matcom.2004.08.002.

[7] M. Levy, Parabolic Equation Methods for Electromagnetic Wave Propagation, IET editions, 2000. doi:10. 1049/pbew045e.

[8] R. H. Hardin, F. D. Tappert, Applications of the split-step Fourier method to the numerical solution of nonlinear and variable coefficient wave equations, SIAM Review 15 (1973) 423-429.

[9] G. D. Dockery, J. R. Kuttler, An improved impedance-boundary algorithm for Fourier split-step solutions of the parabolic wave equation, IEEE Transactions on Antennas and Propagation 44 (1996) 1592-1599. doi: $10.1109 / 8.546245$

[10] A. E. Barrios, A terrain parabolic equation model for propagation in the troposphere, IEEE Transactions on Antennas and Propagation 42 (1994) 90-98. doi:10.1109/8.272306.

[11] D. J. Donohue, J. R. Kuttler, Propagation modeling over terrain using the parabolic wave equation, IEEE Transactions on Antennas and Propagation 48 (2000) 260-277. doi:10.1109/8.833076.

[12] R. Janaswamy, A curvilinear coordinate-based split-step parabolic equation method for propagation predictions over terrain, IEEE Transactions on Antennas and Propagation 46 (1998) 1089-1097. doi:10.1109/8.704813.

[13] W. L. Siegmann, G. A. Kriegsmann, D. Lee, A wide-angle three-dimensional parabolic wave equation, Journal of the Acoustical Society of America 78 (1985) 659-664. doi:10.1121/1.392434.

[14] F. Sturm, J. A. Fawcett, On the use of higher-order azimuthal schemes in 3-D PE modeling, Journal of the Acoustical Society of America 113 (2003) 3134-3145. doi:10.1121/1.1572138.

[15] F. Sturm, Numerical study of broadband sound pulse propagation in three-dimensional oceanic waveguides, Journal of the Acoustical Society of America 117 (2005) 1058-1079. doi:10.1121/1.1855791.

[16] M. Belonosov, M. Dmitriev, V. Kostin, D. Neklyudov, V. Tcheverda, An iterative solver for the 3D Helmholtz equation, Journal of Computational Physics 345 (2017) 330-344. doi:10.1016/j.jcp.2017.05.026.

[17] A. A. Zaporozhets, M. F. Levy, Bistatic RCS calculations with the vector parabolic equation method, IEEE Transactions on Antennas and Propagation 47 (1999) 1688-1696. doi:10.1109/8.814948.

[18] R. Janaswamy, Path loss predictions in the presence of buildings on flat terrain: A 3-D vector parabolic equation approach, IEEE Transactions on Antennas and Propagation 51 (2003) 1716-1728. doi:10.1109/tap.2003. 815415 .

[19] A. Ginestet, Modélisation de la propagation d'une onde electromagnétique sur des scènes de grande taille par résolution de l'equation parabolique 3D vectorielle, Ph.D. thesis, Université Paul Sabatier-Toulouse III (2007).

[20] H. Zhou, A. Chabory, R. Douvenot, Comparisons of discrete and continuous propagators for the modelling of low tropospheric propagation, in: 11th European Conference on Antennas and Propagation (EuCAP), 2017, pp. 1236-1238. doi:10.23919/EuCAP.2017.7928111.

[21] H. Zhou, Modeling the atmospheric propagation of electromagnetic waves in 2D and 3D using Fourier and wavelet transforms, Ph.D. thesis, Université Toulouse 3 (Apr. 2018).

[22] H. Zhou, A. Chabory, R. Douvenot, A 3-D split-step Fourier algorithm based on a discrete spectral representation of the propagation equation, IEEE Transactions on Antennas and Propagation 65 (4) (2017) 1988-1995. doi: 10.1109/TAP.2017.2671018.

[23] W. C. Chew, Electromagnetic theory on a lattrice, Journal of Applied Physics 75 (1994) 4843-4850. doi: 10.1063/1.355770.

[24] F. L. Teixeira, W. C. Chew, Lattice electromagnetic theory from a topological viewpoint, Journal of Mathematics and Physics 40 (1999) 169-187. doi:10.1063/1.532767. 
[25] P. D. Einziger, S. Raz, M. Shapira, Gabor representation and aperture theory, Journal of the Optical Society of America A, Optics and Image Science 3 (4) (1986) 508-522. doi:10.1364/josaa.3.000508.

[26] D. Lugara, C. Letrou, A. Shlivinski, E. Heyman, A. Boag, Frame-based Gaussian beam summation method: Theory and applications, Radio Science 38 (2003) 27-1 - 27-15. doi:10.1029/2001rs002593.

[27] J. J. Maciel, L. B. Felsen, Systematic study of fields due to extended apertures by Gaussian beam discretization, IEEE Transactions on Antennas and Propagation 37 (1989) 884-892. doi:10.1109/8.29383.

[28] J. J. Maciel, L. B. Felsen, Discretized Gabor-based beam algorithm for time-harmonic radiation from twodimensional truncated planar aperture distributions .I. Formulation and solution, IEEE Transactions on Antennas and Propagation 50 (2002) 1751-1759. doi:10.1109/TAP.2002.807419.

[29] A. Chabory, J. Sokoloff, S. Bolioli, Novel Gabor-based Gaussian beam expansion for curved aperture radiation in dimension two, Progress In Electromagnetics Research 58 (2006) 171-185. doi:10.2528/pier05090702.

[30] S. Mallat, A Wavelet Tour of Signal Processing, Academic press, 1999.

[31] B. Z. Steinberg, Y. Leviatan, On the use of wavelet expansions in the method of moments (EM scattering), IEEE Transactions on Antennas and Propagation 41 (1993) 610-619. doi:10.1109/8.222280

[32] T. K. Sarkar, Salazar-Palma, M. C. Wicks, Wavelet Application in Engineering Electromagnetics, Artech House, 2002.

URL http://us .artechhouse.com/Wavelet-Application-in-Engineering-Electromagnetics-P527 .aspx

[33] J. Fröhlich, K. Schneider, An adaptive wavelet-vaguelette algorithm for the solution of PDEs, Journal of Computational Physics 130 (1997) 174-190. doi:10.1006/jcph.1996.5573.

[34] M. Holmström, Solving hyperbolic PDEs using interpolating wavelets, SIAM Journal on Scientific Computing 21 (1999) 405-420. doi:10.1137/s1064827597316278.

[35] T.-K. Hong, B. L. N. Kennett, On a wavelet-based method for the numerical simulation of wave propagation, Journal of Computational Physics 183 (2002) 577-622. doi:10.1006/jcph.2002.7202.

[36] A. Iqbal, V. Jeoti, A split step wavelet method for radiowave propagation modelling in tropospheric ducts, in: IEEE International on RF and Microwave Conference (RFM), 2011, pp. 67-70. doi:10.1109/rfm.2011. 6168697 .

[37] A. Iqbal, V. Jeoti, Numerical modeling of radio wave propagation in horizontally inhomogeneous environment using split-step wavelet method, in: 4th International Conference on Intelligent and Advanced Systems (ICIAS), 2012, pp. 200-205. doi:10.1109/icias.2012.6306187.

[38] S. Mallat, A theory for multiresolution signal decomposition: the wavelet representation, IEEE Transactions on Pattern Analysis and Machine Intelligence 11 (1989) 674-693. doi:10.1515/9781400827268.494

[39] S. Mallat, Multiresolution approximations and wavelet orthonormal bases of $\mathrm{L}^{2}(\mathrm{R})$, Transactions of the American Mathematical Society 315 (1) (1989) 69-87. doi:10.2307/2001373.

[40] M. Frigo, S. G. Johnson, The design and implementation of FFTW3, Proceedings of the IEEE 93 (2005) 216-231.

[41] G. A. Deschamps, Gaussian beam as a bundle of complex rays, Electronics Letters 7 (1971) 684-685. doi: 10.1049/el:19710467. 